\title{
Aneurismas de carótida extracraneal: técnicas quirúrgicas y endovasculares. A propósito de 4 casos
}

\author{
Extracranial carotid aneurisms: surgical and endovascular techniques. Report of 4 cases \\ Oscar Talledo ${ }^{1, a}$, María Alejandra Mena ${ }^{1, \mathrm{~b}}$, Walter Durand ${ }^{2, \mathrm{c}}$, Felipe Plaza ${ }^{2, \mathrm{~d}}$, Oscar De la Peña ${ }^{1, \mathrm{~b}}$, Lourdes \\ Torres $^{1, e}$
}

\section{RESUMEN}

Se presentan 4 casos de aneurismas o pseudoaneurismas de la arteria carótida interna extracraneal. A diferencia de la estenosis carotídea, esta patología es poco frecuente; por lo que las recomendaciones en cuanto al diagnóstico y tratamiento no son uniformes y aun están en discusión, representando así un reto para los médicos de la especialidad. Cada uno de los casos descritos fue tratado de forma particular a las características de anatomía, forma de presentación y riesgos de cada paciente. Los casos fueron de sexo masculino y con un rango etario desde 24 años hasta 77 años de edad. Dos de ellos fueron tratados de forma quirúrgica convencional y dos de forma endovascular. Se presenta las opciones de tratamiento quirúrgico y endovascular en nuestro medio en una enfermedad en la que se tiene experiencia limitada en la mayoría de centros médicos a nivel mundial.

PALABRAS CLAVE: Aneurisma, arteria carótida interna/lesiones. (Fuente: DeCS BIREME).

\section{SUMMARY}

We present 4 cases of extracranial internal carotid aneurisms or pseudo aneurisms. Opposite to carotid stenosis, this entity is rare, and recommendations for its diagnosis and treatment are not standardized and subject to controversy. Each of the cases presented here were treated differently. All cases were males ranging in age from 24 to 77 years of age; 2 were treated using conventional surgical methods and the remaining two using an endovascular approach. We present here the surgical options for a rare entity with limited worldwide experience.

KEYWORDS: Aneurysms, carotid artery/injuries, internal. (Source: MeSH NLM).

\section{INTRODUCCIÓN}

Los aneurismas extracraneales de la arteria carótida interna son extremadamente infrecuentes, y se estima que la mitad de ellos son en realidad pseudoaneurismas asociados a trauma, infección o aneurismas anastomóticos (1). El conocimiento que tenemos hoy en día al respecto proviene de reportes o series de casos, por lo que es difícil establecer guías y estándares de tratamiento. Hoy contamos con técnicas terapéuticas endovasculares que se añaden a las técnicas quirúrgicas convencionales para incrementar nuestras herramientas terapéuticas en este campo; sin embargo, se conoce poco sobre los resultados a

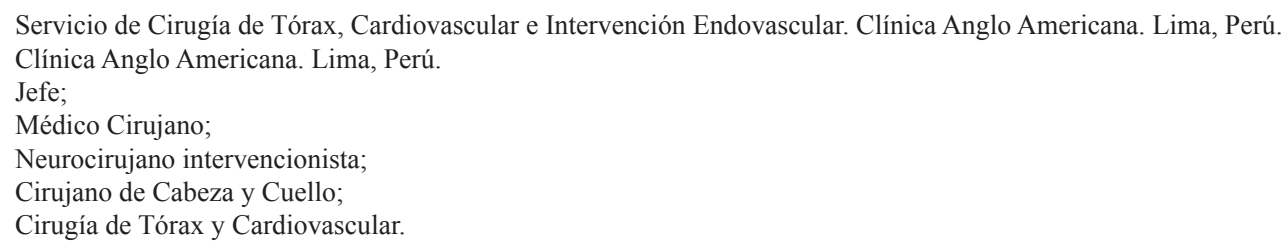


mediano y largo plazo tanto del tratamiento quirúrgico convencional como del endovascular (2-5).

Se presentan cuatro diferentes casos, tres de pseudoaneurismas y uno de aneurisma de la carótida interna extracraneal tratados tanto con cirugía abierta como cirugía endovascular, demostrando las diferentes posibilidades de tratar esta patología.

\section{PRESENTACIÓN DE CASOS}

\section{Caso 1}

Varón de 24 años ingresó al servicio de emergencia, trasladado de Pucallpa (ciudad de la selva), con historia de haber recibido múltiples disparos de cartucho de perdigones en cabeza, cuello y tórax, aproximadamente 3 días antes. Al examen clínico inicial se diagnosticó hemo-neumotórax izquierdo el cual fue drenado; además, presentó un proyectil metálico en el septo interventricular cardíaco el cual se manejó con tratamiento conservador. Se resecaron múltiples proyectiles metálicos redondeados en cara, cuero cabelludo, cuello, tórax y muslo. Al examen cervical izquierdo presentaba una tumoración y equimosis con thrill a la palpación y soplo sistodiastólico a la auscultación (Figura 1A). Alrededor de la tumoración había hasta 3 heridas de perdigón. Una ecografía confirmó el diagnóstico de pseudoaneurisma de la arteria carótida interna izquierda (ACII) y una arteriografía determinó que el pseudoaneurisma era de $7 \mathrm{x} 4 \mathrm{~mm}$ (Figura 1C), localizado a aproximadamente 4 $\mathrm{cm}$ distal a la bifurcación de la carótida, siendo los diámetros de la arteria carótida distal y proximal de $5,1 \mathrm{~mm}$ y $6,2 \mathrm{~mm}$ respectivamente, por lo cual se colocó un stent recubierto (Stent-graft) Viabahn de $6 \times 25 \mathrm{~mm}$ en una segunda intervención ingresando por acceso femoral, logrando exclusión total del aneurisma al cubrir la perforación en el segmento 2 de la ACII con el stent graft (6) (Figuras 1B y 1D). El paciente tuvo evolución favorable y fue dado de alta sin inconvenientes.

\section{Caso 2}

Varón de 38 años que ingresó al servicio de emergencia con hemiparesia izquierda y afasia de reciente presentación. La resonancia magnética

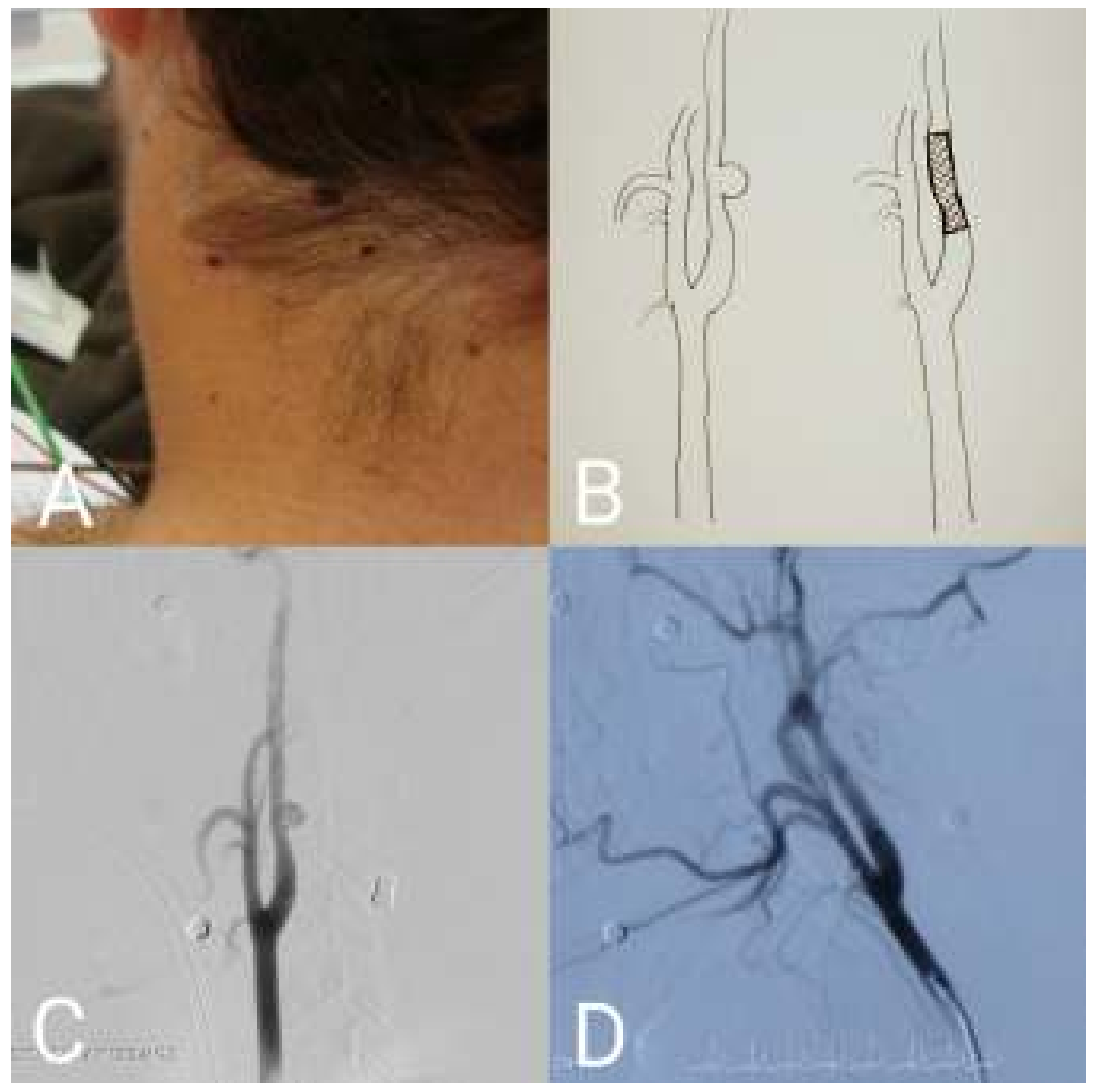

Figura 1. A: Tumoración pulsátil posterolateral cervical izquierda. B: Esquema de hallazgo y tratamiento endovascular realizado. C: Angiografía diagnóstica muestra carótida común izquierda con pseudoaneurisma de la ACII. D: Angiografía de carótida común izquierda post intervención endovascular. En las fotos C y D también se pueden observar los objetos metálicos redondeados (perdigones). 
(RMN) mostró microinfarto debido a probable microembolismo en la corteza del hemisferio derecho. El paciente era conocido del servicio ya que dos años antes había sido evaluado por una tumoración cervical pulsátil debida a un pseudoaneurisma de la arteria carótida interna derecha (ACID) de aproximadamente $5 \mathrm{~cm}$ de diámetro que no se quiso tratar. Su historia pasada era de herida de bala cervical derecha a los 12 años que recibió tratamiento quirúrgico en otra institución. Diez días antes de su ingreso tuvo accidente isquémico transitorio (TIA) con hemiparesia izquierda a predominio de miembro inferior y RMN encefálica normal. El día de ingreso fue evaluado por el neurólogo y se realizó nueva resonancia magnética cervical que mostró pseudoaneurisma en ACID de $30 \mathrm{~mm}$ con presencia de coágulo en la pared. La angiotomografía cervical confirmó la presencia de pseudoaneurisma de $47 \times 36 \times 38 \mathrm{~mm}$ localizada a $6 \mathrm{~mm}$ distal a la bifurcación y estenosis de $80 \%$ en la ACID proximal al pseudoaneurisma (Figura 2A).

Luego de la evaluación conjunta con el servicio de Neurocirugía intervencionista, se decidió tratamiento endovascular conjunto.
Se realizó una arteriografía por acceso femoral, colocando un catéter guía en la arteria carótida común derecha (ACCD), luego un filtro anti embólico en ACID distal, seguidamente se posicionó un wallstent cerrado desde la carótida interna hasta el tronco común, y a través del catéter guía se colocó un micro catéter paralelo introducido al pseudoaneurisma. Inicialmente se desplegó parcialmente el wallstent y se colocaron coils intra aneurismáticos (número aproximado 23), luego se desplegó completamente el stent metálico, al mostrar la arteriografía oclusión parcial del pseudoaneurisma, se decidió colocar un balón de angioplastia en el origen de la ACID con el objeto de impactar el stent venciendo la estenosis proximal y a la vez observar el libre pasaje de contraste hacia la carótida externa. Logrado esto, se reemplazó el balón de angioplastia por un stent recubierto de $5 \times 22 \mathrm{~mm}$ Advanta V12 dentro del wallstent colocado previamente en este mismo procedimiento.

De esta manera se cubrió las paredes de la ACID desde su origen en la bifurcación, ocluyendo la entrada del pseudoaneurisma en su totalidad (Figura 2B). Con esta maniobra se logró excluir la entrada al

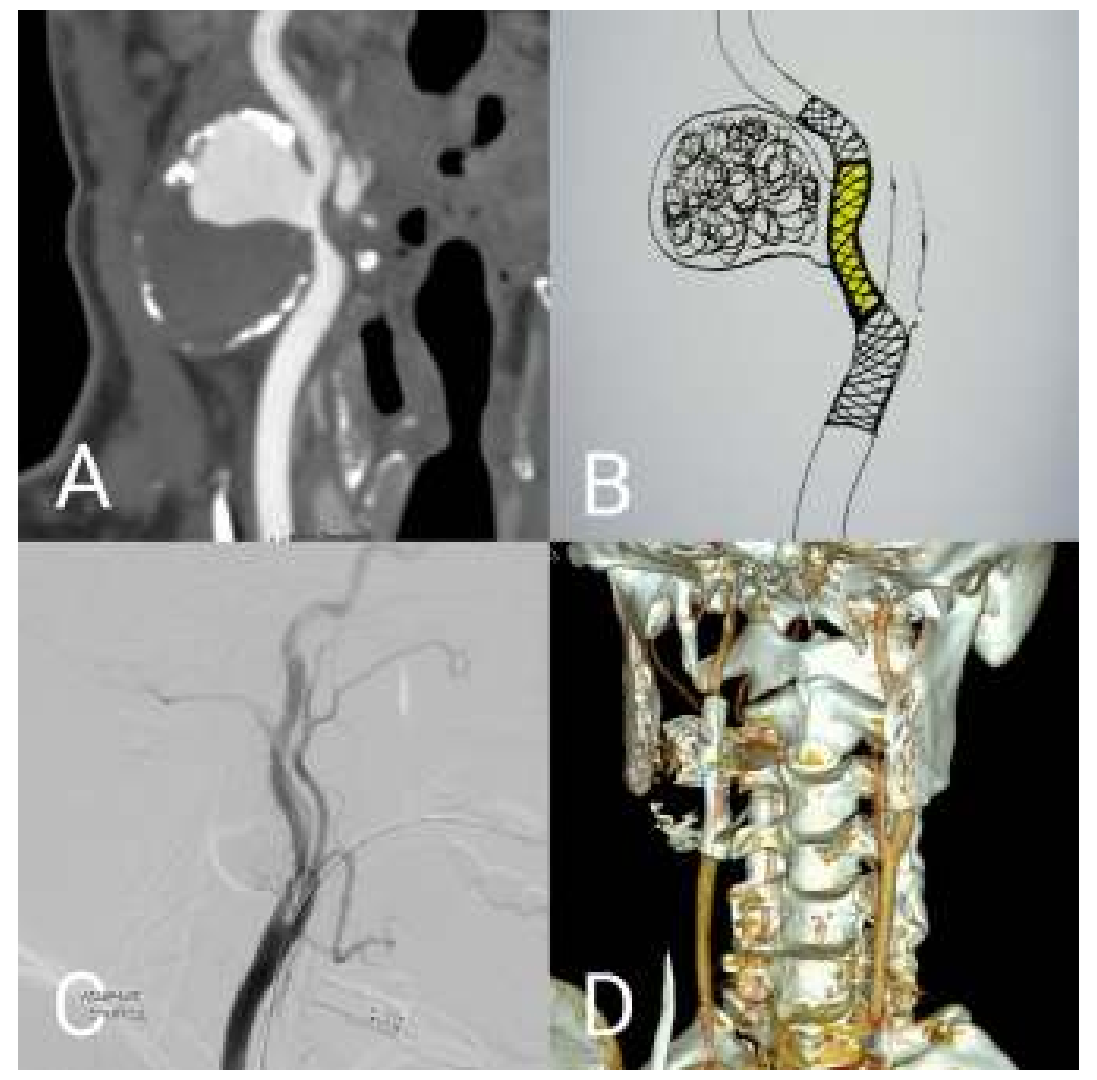

Figura 2. A: Angiotomografía cervical que muestra pseudoaneurisma de ACID. B: Esquema de tratamiento endovascular realizado, coils dentro de aneurisma, luego stent recubierto (en amarillo) dentro del stent desnudo. C: Angiografía post intervención endovascular que muestra flujo normal en ACID y ACED, así como exclusión total del pseudoaneurisma. D: Angiotomografía en el seguimiento 1 mes post intervención. 
pseudoaneurisma y la arteriografía post intervención mostró exclusión total del pseudoaneurisma con pasaje ininterrumpido a través de ambas carótidas interna y externa (Figura 2C).

El paciente ha tenido seguimiento clínico y tomográfico (Figura 2D) por un año, sin recurrencia de síntomas ni secuelas neurológicas y con persistencia de exclusión del pseudoaneurisma.

\section{Caso 3}

Varón 67 años, gran fumador, que ingresó al servicio de emergencia con 20 horas de enfermedad caracterizada por amaurosis del ojo derecho de inicio súbito. La evaluación oftalmológica concluyó que se trataba de una isquemia de retina. Se realizó eco Doppler de carótidas que mostró la presencia de un trombo flotante en la ACID (Figura 3A). La angiotomografía mostró una dilatación aneurismática de la arteria carótida común derecha (ACCD) que se extendía a la ACID (Figura 3B).
Se decidió intervención quirúrgica de emergencia en la cual, al abrir el aneurisma, se observó una úlcera penetrante ocupada por un trombo que emergía de ella con una cola libre, el cual fue resecado (Figura 3C).

Para el tratamiento del aneurisma se realizó incisión de aneurismotomía anterior longitudinal, luego de identificar la úlcera y el trombo ocupante en la pared posterior del aneurisma, se resecó, con una incisión en losange, incluyendo la úlcera y el trombo; luego se cerró la pared posterior con sutura lineal. Finalmente se realizó la aneurismorrafia, para lo cual se recortaron los bordes de la incisión de la pared anterior, y se cerró primariamente con sutura lineal recuperando el diámetro original de la ACCD y ACID proximal (Figura 3D).

\section{Caso 4}

Varón 77 años que se presentó con foliculitis recurrente en la zona de una cicatriz de endarterectomía de carótida interna izquierda realizada tres años antes. La foliculitis fue tratada con antibióticos y drenaje

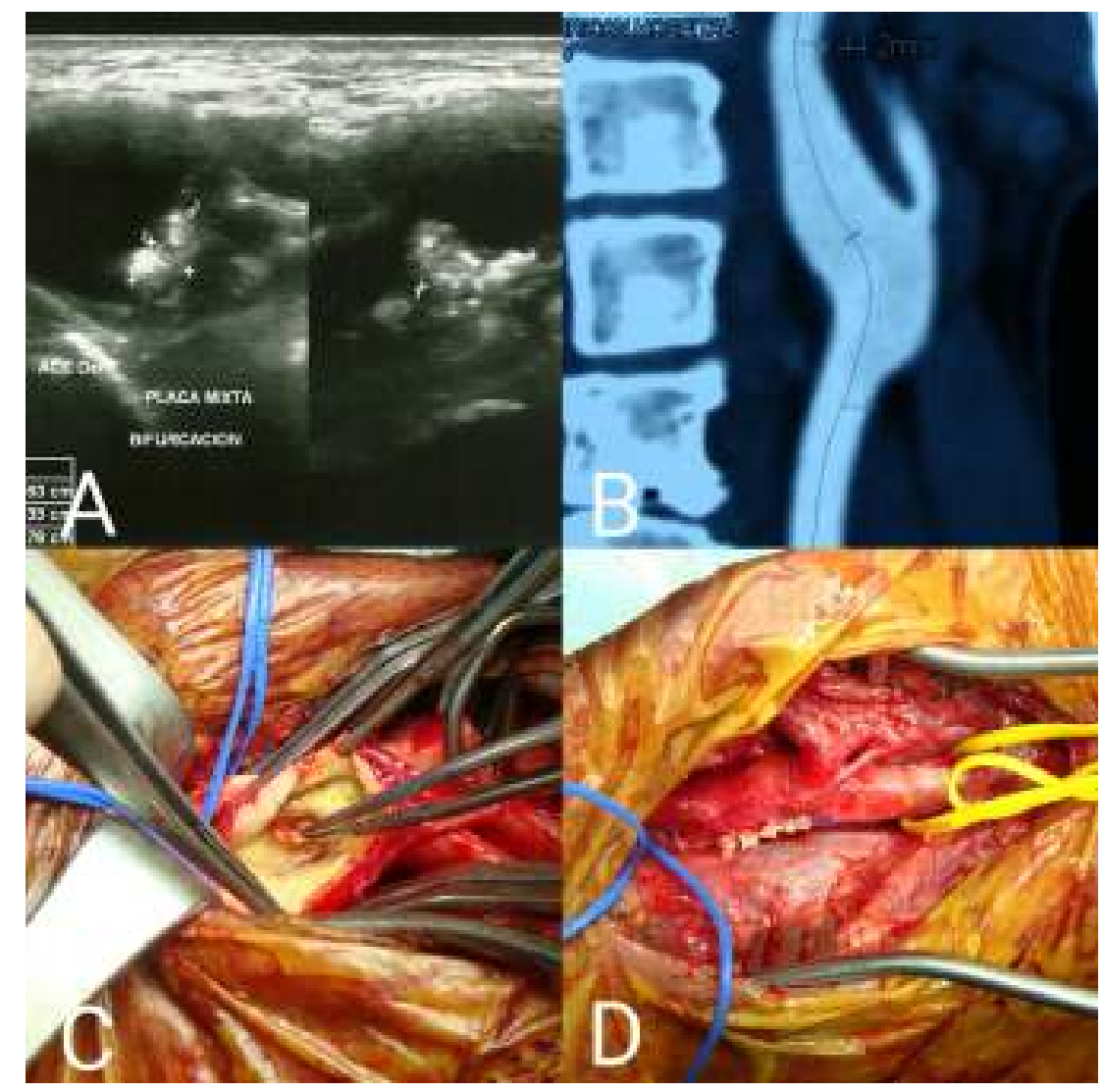

Figura 3. A: Ecografía de carótida en la que se evidencia presencia de trombo intraluminal flotante en lesión aneurismática. B: Angiotomografía cervical que muestra la misma lesión de aneurisma, pero sin evidenciar trombo intraluminal o placa. C: Hallazgo intraoperatorio dentro de aneurisma: placa ateromatosa ulcerada con contenido de trombo flotante y fibrina. D: Cierre lineal de aneurismorrafia 
de absceso subcutáneo en el servicio de cirugía de cabeza y cuello, a pesar de lo cual presentó recurrencia adquiriendo forma de una fístula cutánea con drenaje de sangre con pus a pesar de tratamiento.

Se planteó entonces el diagnóstico de pseudoaneurisma infectado que se comprobó con una ecografía. Debido al alto riesgo de ruptura y muerte por sangrado o septicemia se decidió tratamiento inmediato.

Dado que, tres años antes se había realizado una endarterectomía con colocación de parche de dacrón a manera de arterioplastía, se hacía necesario obtener control proximal y distal previo a la exposición del pseudoaneurisma. Se evaluó el caso con el servicio de cirugía de cabeza y cuello, decidiéndose tratamiento quirúrgico en equipo conjunto.

Se ingresó con una incisión desde la zona pre auricular (Figura 4D) y se logró obtener control de la ACII distal al pseudoaneurisma, luego de seccionar el músculo digástrico. Luego se realizó la incisión proximal muy cerca a la escotadura esternal obteniéndose control de la ACCI proximal al pseudoaneurisma. Luego de heparinizar, se colocaron clamps proximal y distal. Se ingresó al aneurisma y obtuvo control también de la arteria carótida externa izquierda (ACEI) con catéter balón Fogarty (Figura 4B). Se inició la disección del pseudoaneurisma, el cual se resecó completamente con el parche de dacrón dehiscente adherido y se incluyó en la resección la parte distal de la ACCI y su bifurcación hasta encontrar en ambos extremos arteria sana. El pseudoaneurisma fue resecado en bloque con la fístula de piel incluida (Figura 4A). Luego del lavado y resección de todo el tejido necrótico infectado se decidió revascularizar la carótida con bypass de vena safena reversa autóloga desde la arteria carótida común a la interna con anastomosis término terminal y reimplante de la arteria carótida externa en forma termino lateral al injerto de vena (Figura 4C). La evolución en el postoperatorio fue sin complicaciones.

Al tercer año después de la operación presentó un TIA, detectándose oclusión del bypass de vena. Actualmente tiene 8 años de seguimiento y no tiene recurrencia de infección, síntomas, ni secuela neurológica.

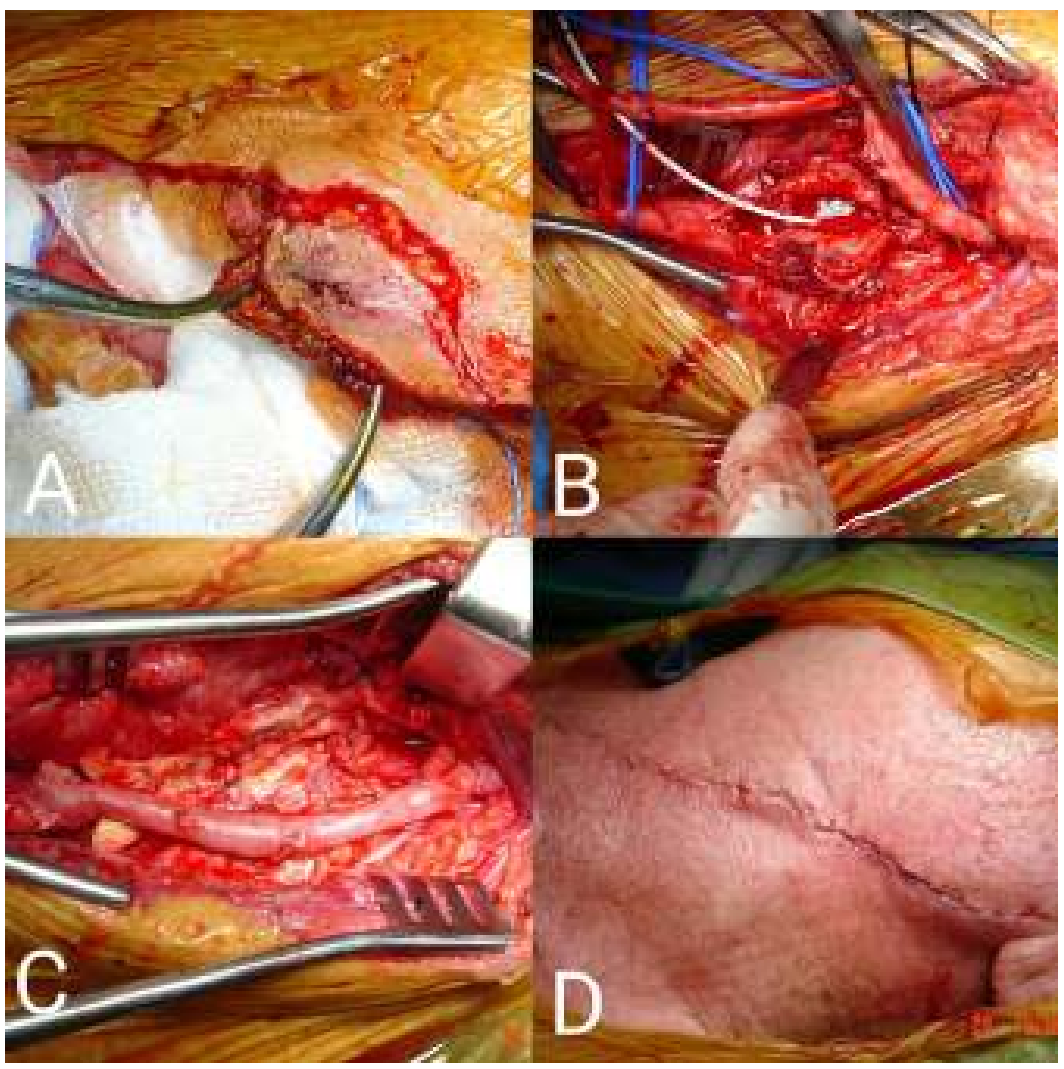

Figura 4. A: Fistula cutánea. B: Pseudoaneurisma abierto, uso de catéter Fogarty para control de arteria carótida externa. C: Bypass con injerto de vena safena reversa autóloga de ACCI a ACII con reimplante de ACEI. D: Herida operatoria cerrada que se extiende desde la zona pre auricular hasta zona supraclavicular. 


\section{DISCUSIÓN}

Los reportes de aneurismas y pseudoaneurismas carotideos están agrupados en la misma patología, dada la poca frecuencia y similitud de ambos. Sin embargo la proporción y prevalencia de los pseudoaneurismas y aneurismas verdaderos varía en las diferentes series grandes publicadas (5,7-9), apareciendo los pseudoaneurismas como más frecuentes.

En el 2000, El-Sabrout y Cooley (5), publicaron la serie de casos del Instituto del corazón de Texas (THI); encontraron 67 casos de aneurismas carotideos extracraneales, 38 de los cuales fueron pseudoaneurismas, todos ellos estuvieron asociados a endarterectomia previa. Así mismo, hacen una revisión de las series previas publicadas en la literatura, encontrando información desde 1976, resaltando que es escasa la información sobre el manejo de esta patología, incluso se menciona que algunos de los centros más grandes del mundo reportan no más de 1 caso por año.

Hoy en día la serie más grande, fue publicada en 2015 por el grupo de la Clínica Mayo, y consta de 141 casos encontrados en 14 años (9). La mayoría fueron pseudoaneurismas $(82 \%)$ y el resto (18\%) fueron verdaderos, la mitad eran asintomáticos. Para el manejo se usó: intervención con cirugía, con intervención endovascular, y manejo no operatorio. El manejo no operatorio fue más común en los asintomáticos, ocurrió aproximadamente en la mitad de casos y al seguimiento de 77 meses no se observó morbilidad o mortalidad relacionada al aneurisma ni requerimiento de intervención (9).

Los aneurismas y pseudoaneurismas presentan riesgo de ruptura y embolización. Los infectados pueden además causar sepsis o abscesos distales $(10,11)$.

El aneurisma carotideo puede ser tratado con cirugía abierta convencional o con métodos endovasculares, utilizando stents, stent recubiertos o coils. Debido a las características y restricciones de la localización anatómica y de la importancia del territorio irrigado, la mayoría de los autores concluyen que esto representa un reto, tanto desde el punto de vista técnico de las intervenciones como de la toma de decisión de manejo.

Cada caso debe ser evaluado individualmente para elegir el mejor método terapéutico. En el caso 1 la entrada al pseudoaneurisma producido PAF en la arteria carótida interna era muy distal para obtener control por cirugía, es por eso que se decidió la colocación de un stent recubierto.

En el caso 2 el pseudoaneurisma era muy grande $(5 \mathrm{~cm})$ y con presencia de cicatriz y adherencias de una cirugía previa. Los riesgos de una cirugía abierta eran muy altos y se eligió terapia endovascular. Al principio con coils y stent desnudo, pero al no lograr la exclusión completa se agregó un stent recubierto dentro del stent con el cuidado de cerrar solo la entrada al pseudoaneurisma, pero dejando permeable el pasaje a la AC externa. Los casos 3 y 4 fueron tratados con éxito con cirugía abierta con técnicas adaptadas para cada patología particular.

En el caso 3 es necesario resaltar el hecho de que la ecografía descubrió con claridad la presencia de un trombo flotante (Figura 3A) que no se evidenció en la angiotomografía (Figura 3B), y que durante la cirugía se confirmó la presencia de una úlcera con dicho trombo flotante (Figura 3C). Esto nos hace reflexionar sobre la cantidad de veces en que no se puede diagnosticar la fuente de la embolia porque el método de angiotomografía no es suficientemente sensible y no lo permite, mientras que la ecografía que si es sensible, no siempre es aplicable a todo territorio arterial.

En conclusión, ante un pseudoaneurisma/ aneurisma de carótida extracraneal la cirugía abierta sigue siendo el tratamiento indicado como primera opción. Cuando el riesgo es alto debido a cirugía o radiación previa, inaccesibilidad debido a localización distal, presencia de traqueostomia, lesión del nervio laríngeo recurrente contralateral, mal estado general o fragilidad del paciente, entonces puede considerarse la opción endovascular.

El método endovascular utilizando coils o stent recubierto puede ser realizado eficientemente con resultados exitosos y bajas complicaciones. Los resultados a largo plazo deberán ser evaluados.

\section{Contribución de autoría:}

OT: Cirujano tratante de los 4 casos, revisión; MM: Redacción, Búsqueda bibliográfica; WD: Cirujano tratante en conjunto en caso 2, revisión; FP: Cirujano tratante en conjunto en caso 4, revisión; OD: Redacción, Búsqueda de datos; LT: Revisión, Edición 


\section{Declaración de financiamiento y de conflictos de intereses:}

Los autores declaran no haber recibido apoyo económico en ninguna de sus formas (subvención, ni donación). Asimismo, declaramos que no existen conflictos de intereses del autor o autores.

\section{Correspondencia:}

María Alejandra Mena

Correo electrónico:m.mena@vascor.pe

Teléfono: 958879053

\section{REFERENCIAS BIBLIOGRÁFICAS}

1. Atkins MD, Bush, Ruth L. Carotid Artery: Aneurysms. En:Cronenwett J, Johnston K. Rutherford's Vascular Surgery. 8th ed. London: Saunders Elsevier; 2014.p. 1583 - 97.

2. Terramani TT, Workman MJ, Loberman Z, et al. Adjunctive endovascular techniques in the management of postoperative carotid artery pseudoaneurysms--useful armamentarium for vascular surgeons--three case reports. Vascular and endovascular surgery. 2003; 37(3):207-12.

3. Gupta R, Thomas AJ, Masih A, Horowitz MB. Treatment of extracranial carotid artery pseudoaneurysms with stent grafts: case series. Journal of neuroimaging. 2008; 18(2):180-3.
4. Pulli R, Dorigo W, Pratesi G, Fargion A, Pratesi C. Single-center experience on endovascular repair of noninfected extracranial internal carotid artery pseudoaneurysms. Annals of vascular surgery. 2013; 27(5):672 e13-7.

5. El-SabroutR,CooleyDA.Extracranial carotidartery aneurysms:TexasHeartInstitute experience. Journal of vascular surgery. 2000; 31(4):702-12.

6. Bouthillier A, van Loveren HR, Keller JT. Segments of the internal carotid artery: a new classification. Neurosurgery. 1996; 38(3):425-32.

7. Zhou W, Lin PH, Bush RL, et al. Carotid artery aneurysm: evolution of management over two decades. Journal of vascular surgery. 2006; 43(3):493-

6.

8. RossetE,AlbertiniJN,MagnanPE,EdeB,Thomassin JM,BranchereauA. Surgical treatmentofextracranial internal carotid artery aneurysms. Journal of vascular surgery. 2000; 31(4):713-23.

9. Fankhauser GT, Stone WM, Fowl RJ, et al. Surgical and medical management of extracranial carotid artery aneurysms. Journal of vascular surgery. 2015; 61(2):389-93.

10. Baril DT, Ellozy SH, Carroccio A, Patel AB, Lookstein RA, Marin ML. Endovascular repair of aninfectedcarotidarterypseudoaneurysm. Journalof vascular surgery. 2004; 40(5):1024-7.

Recibido: 09/01/2017

Aceptado: 29/08/2017 